\title{
Cytokeratin 5/6 and cytokeratin 8/18 expression in triple negative breast cancers: clinicopathologic significance in South-Asian population
}

\author{
Atif Ali Hashmi ${ }^{1}$, Samreen Naz ${ }^{1}$, Shumaila Kanwal Hashmi ${ }^{2}$, Zubaida Fida Hussain ${ }^{1}$, Muhammad Irfan ${ }^{1}$, \\ Syed Muhammad Abu Bakar', Naveen Faridi ${ }^{1}$, Amir Khan ${ }^{3^{*}}$ (D) and Muhammad Muzzammil Edhi ${ }^{4}$
}

\begin{abstract}
Objective: Cytokeratin 5/6 and Cytokeratin 8/18 are basal and luminal markers of breast cancer and they have pathological and prognostic significance in breast cancer. We performed Cytokeratin 5/6 and CK8/18 immunohistochemistry on 150 cases of triple negative breast cancers and association with various clinicopathological features was evaluated.

Results: Positive CK5/6 expression was noted in 8\% (12 cases) of TNBC while 2.4\% (4 cases) showed focal positive $(<10 \%)$ and $89.3 \%$ (134) were negative with CK5/6. Complete loss of CK8/18 expression was seen in $4.7 \%$ (7 cases) while $32.7 \%$ (49 cases) revealed focal loss of CK8/18 and 62.7\% (94 cases) showed intact normal expression of CK8/18. No significant association of $\mathrm{CK} 5 / 6$ and $\mathrm{CK} 8 / 18$ with various clinicopathological parameters was observed. We found a low expression of basal cytokeratin (CK5/6) in TNBC our studied population, while loss/altered expression of CK8/18 in approximately $38 \%$ of TNBC. Although no prognostic relevance of these finding was noted in our study, however these findings are different from those reported in literature in other parts of the world. Therefore we suggest a more through immunohistochemical and genomic profiling of TNBC in our population for better understanding of this disease in this part of the world.
\end{abstract}

Keywords: Triple negative breast cancer, CK5/6, CK8/18, Basal cytokeratins, Luminal cytokeratins

\section{Introduction}

Triple negative breast cancer (TNBC) is a heterogeneous group of breast cancer accounting for $15-20 \%$ of newly diagnosed breast cancer [1-3]. TNBC is a clinically defined term with lack of immunohistochemistry (IHC) expression of Estrogen receptor (ER), progesterone receptor (PR) and human epidermal growth factor receptor 2 (her2neu) according to College of American Pathologist (CAP)/ASCO guidelines [4, 5]. This clinically defined subtype of breast cancer also comprise basal like molecular subtype, however triple negative and basal

*Correspondence: dramirkhan04@gmail.com

${ }^{3}$ Kandahar University, Kandahar, Afghanistan

Full list of author information is available at the end of the article breast cancers are not synonymous and there is substantial overlap and heterogeneity among these two groups. For example in a study; $71 \%$ of TNBC were found to be of basal subtype and conversely $77 \%$ basal tumors were triple negative by IHC analysis [6]. However, in resource limited countries like Pakistan where molecular testing is not widely available, relying on IHC expression of cytokeratins can be considered acceptable. Basal like breast cancers usually express basal cytokeratins like CK5/6, CK14 and CK17 [7]. CK5/6 is the most useful and most important marker for the identification of basal subgroup of TNBC [8]. CK8/18 is another marker which can be called luminal marker as it represents normal luminal epithelial differentiation [8] and thus loss of CK8/18 expression in TNBC may have pathological 
relevance. Expression of these markers in TNBC has not been widely studied in our population, therefore in the present study we aimed to evaluate the expression of CK 5/6 and CK8/18 in TNBC in our population and their prognostic significance.

\section{Main text \\ Methods}

Total 150 cases of TNBC were selected from records of pathology department archives. The surgical specimens included modified radical mastectomies, simple mastectomies and wide local excisions with sentinel lymph node dissection. All patients underwent surgeries at Liaquat National hospital, Karachi from January 2008 till December 2013 over a period of 6 years. Study was approved by research and ethical review committee of the institution and informed consent was taken from all patients at the time of surgery. Hematoxylin and eosin stained slides and paraffin blocks of cases were retrieved and new sections were cut where necessary. Slides of all cases were reviewed by two senior histopathologists and pathologic characteristics like histologic type, tumor grade, T-stage, $\mathrm{N}$-stage, lymphovascular invasion, necrosis, fibrosis, lymphocytic infiltration (tumor infiltrating lymphocytes), presence of insitu component, pagetoid spread and dermal lymphatic invasion were evaluated. Clinical records of all patients were also reviewed from institutional records to evaluate patient's age, history of radiation and chemotherapy and recurrence status. Moreover, representative blocks of each case were selected and ER, PR and Her2neu immunohistochemistry were performed to reconfirm triple negative status.

ER, PR, Her2neu and Ki67 IHC were performed using DAKO antibodies as under, with EnVision ${ }^{\mathrm{TM}}$ FLEX, high pH DAKO kit according to manufacturer's protocol.

1. FLEX monoclonal rabbit anti-human estrogen receptor alpha, clone EP1.

2. FLEX monoclonal mouse anti-human progesterone receptor clone PgR 636.

3. Polyclonal rabbit anti-human c-erbB-2 oncoprotein.

4. FLEX monoclonal mouse anti-human Ki67 antigen clone MIB-1.

Nuclear staining of ER and PR in $>1 \%$ of cancer cells was considered positive. For her2neu, cases were scored $1+, 2+$ and $3+$ according to CAP guidelines. For equivocal cases $(2+)$, FISH was performed using Path Vysion Her2neu probe kit according to manufacturer's protocols. Results were expressed as ratio of her2neu signals as compared to CEP17 signals according to ASCO/CAP guidelines.
Ki67 immunostaining was recorded as continuous variables based on proportion of positive stained cancer cells. Furthermore on the basis of Ki67 index, cases were categorized into $<15,16-24,25-44$ and $>44 \%$ ki67 index categories.

CK5/6 IHC was performed by using FLEX Monoclonal Mouse Anti-human Cytokeratin 5/6, clone D5/16 B4 by DAKO envision method according to manufacturers protocol. Intermediate to strong cytoplasmic and membranous staining in more than $10 \%$ cells was considered positive. Weak to intermediate staining in $<10 \%$ was taken as focal positive, while no staining was considered as negative (Additional file 1: Figure S1).

CK 8/8 IHC was performed using monoclonal mouse anti-human Cytokeratin 5/6, clone D5/16 B4, according to DAKO envision method. Intermediate to strong cytoplasmic and membranous staining in more than $50 \%$ cells was considered positive. Weak to intermediate staining (cytoplasmic, with loss of membrane reactivity) in $10-50 \%$ was taken as focal positive, while no staining or weak staining in $<10 \%$ was considered as negative (Additional file 1: Figure S1).

Recurrence status and follow-up were evaluated by reviewing hospital medical record. Overall survival was taken as time from surgical excision till death or last follow-up and disease free survival was defined as time between surgical excision and local recurrence or distant metastasis, death or last follow-up.

Statistical package for social sciences (SPSS 21) was used for data compilation and analysis. Mean and standard deviation were calculated for quantitative variables. Frequency and percentage were calculated for qualitative variables. Chi square was applied to determine association. Student $t$ test or Mann whitney test were applied to compare difference in means among groups. $P$ value $\leq 0.05$ as significant. Survival curves were plotted using Kaplan-Meier method and the significance of difference between survival curves were determined using log-rank ratio. P-value $\leq 0.05$ was taken as significant.

\section{Results}

Mean age of the patients involved in the study was 48.9 years and most common age group was $31-50$ years. Most of the patients presented at stage T2 with a high mean ki67 index i.e. $46.9 \%$. $42.7 \%$ of cases had nodal metastasis. Although $84 \%$ cases were of conventional invasive ductal carcinoma, NST; however a significant proportion of cases were of metaplastic histology (9.3\%). Majority cases were of high grade ( $86.7 \%$ grade III). Most tumors show lymphocytic infiltration and necrosis. Most of the tumors lack insitu component (61\%). Local recurrence or late distant metastasis was noted in $17.8 \%$ of cases (Table 1). 
Table 1 Descriptive statistics of studied population

\begin{tabular}{|c|c|}
\hline & n (\%) \\
\hline Age (years) ${ }^{\mathrm{a}}$ & $48.85 \pm 11.49$ \\
\hline \multicolumn{2}{|l|}{ Age groups (years) } \\
\hline$\leq 30$ & $5(3.3)$ \\
\hline $31-50$ & $84(56)$ \\
\hline$>50$ & $61(40.7)$ \\
\hline Tumor size (unit) ${ }^{\mathrm{a}}$ & $4.01 \pm 1.99$ \\
\hline \multicolumn{2}{|l|}{ Tumor stage } \\
\hline $\mathrm{T} 1$ & $26(17.3)$ \\
\hline $\mathrm{T} 2$ & $79(52.7)$ \\
\hline $\mathrm{T} 3 / \mathrm{T} 4$ & $45(30)$ \\
\hline Ki67 index (\%) & $46.89 \pm 23.88$ \\
\hline \multicolumn{2}{|l|}{ ki67 index groups (\%) } \\
\hline$\leq 15$ & $17(11.3)$ \\
\hline $16-24$ & $8(5.3)$ \\
\hline $25-44$ & $45(30)$ \\
\hline$>44$ & $80(53.3)$ \\
\hline \multicolumn{2}{|l|}{ Nodal status } \\
\hline Positive & $64(42.7)$ \\
\hline Negative & $86(57.3)$ \\
\hline \multicolumn{2}{|l|}{ Nodal stage } \\
\hline No & $88(58.7)$ \\
\hline $\mathrm{N} 1$ & $30(20)$ \\
\hline N2 & $13(8.7)$ \\
\hline N3 & $19(12.7)$ \\
\hline \multicolumn{2}{|l|}{ Histological subtypes } \\
\hline IDC & $127(84.7)$ \\
\hline Papillary & $6(4)$ \\
\hline Medullary & $1(0.7)$ \\
\hline Metaplastic & $14(9.3)$ \\
\hline Mixed & $2(1.3)$ \\
\hline \multicolumn{2}{|l|}{ Tumor grade } \\
\hline Grade-I & $1(0.7)$ \\
\hline Grade-II & $19(12.7)$ \\
\hline Grade-III & $130(86.7)$ \\
\hline \multicolumn{2}{|l|}{ Lymphocytic infiltration } \\
\hline Absent & $15(10)$ \\
\hline Moderate & $110(73.3)$ \\
\hline Severe & $25(16.7)$ \\
\hline \multicolumn{2}{|l|}{ Lymphovascular invasion } \\
\hline Present & $36(24)$ \\
\hline Absent & $114(76)$ \\
\hline \multicolumn{2}{|l|}{ Dermal lymphatic invasion } \\
\hline Present & $10(6.7)$ \\
\hline Absent & $140(93.3)$ \\
\hline \multicolumn{2}{|l|}{ Type of surgery } \\
\hline Modified radical mastectomy & $94(62.7)$ \\
\hline $\begin{array}{l}\text { Simple mastectomy with sentinel lymph node dissec- } \\
\text { tion }\end{array}$ & $42(28)$ \\
\hline Wide local excision & $14(9.3)$ \\
\hline
\end{tabular}

Table 1 (continued)

\begin{tabular}{ll}
\hline & $\mathbf{n}(\%)$ \\
\hline Necrosis & \\
Absent & $21(14)$ \\
Moderate & $90(60)$ \\
Severe & $39(26)$ \\
Fibrosis & \\
Mild & $42(28)$ \\
Moderate & $88(58.7)$ \\
Severe & $20(13.3)$ \\
Insitu component & \\
Present & $58(38.7)$ \\
Absent & $92(61.3)$ \\
Pagetoid spread & \\
Present & $2(1.3)$ \\
Absent & $148(98.7)$ \\
Perinodal extension & \\
Present & $30(20)$ \\
Absent & $120(80)$ \\
Adjuvant chemotherapy $(\mathrm{n}=101)$ & \\
Yes & $98(97)$ \\
No & $3(3)$ \\
Ydjuvant radiotherapy $(\mathrm{n}=101)$ & \\
No & $69(68.3)$ \\
Reoccurrence $(\mathrm{n}=101)$ & $32(31.7)$ \\
Yes & \\
No & $18(17.8)$ \\
\hline
\end{tabular}

a Mean \pm SD

Positive (>10\%) CK5/6 expression was noted in $8 \%$ (12 cases) of TNBC while $2.4 \%$ (4 cases) showed focal positive $(<10 \%)$ and $89.3 \%$ (134) were negative with CK5/6. Complete loss of CK8/18 expression was seen in $4.7 \%$ (7 cases) while $32.7 \%$ (49 cases) revealed focal loss of CK8/18 and $62.7 \%$ (94 cases) showed intact normal expression of CK8/18. No significant association of CK5/6 and CK8/18 with various clinicopathological parameters was observed (Tables 2 and 3). Similarly no significant association of $\mathrm{CK} 5 / 6$ and $\mathrm{CK} 8 / 18$ with recurrence status of the patients was noted (Additional file 2: Figure S2).

\section{Discussion}

In the present study we assessed expression of one basal $(\mathrm{CK} 5 / 6)$ and one luminal marker (CK8/18) in TNBC in our population and found that; 8 and $2.7 \%$ of TNBC showed positive and focal positive expression of CK5/6 respectively and 4.7 and $32.7 \%$ of TNBC revealed complete loss and focal loss of CK 8/18 expression respectively. In all those cases in which focal loss CK8/18 was 
Table 2 Association of CK 5/6 expression with clinicopathologic features of triple negative breast cancer

\begin{tabular}{|c|c|c|c|c|c|}
\hline & \multicolumn{4}{|l|}{ n (\%) } & \multirow[t]{2}{*}{ P-value } \\
\hline & $\begin{array}{l}\text { No loss } \\
\text { of expression } \\
(n=12)\end{array}$ & $\begin{array}{l}\text { Complete loss } \\
\text { of expression }(n=4)\end{array}$ & $\begin{array}{l}\text { Focal loss } \\
\text { of expression }(n=134)\end{array}$ & Total $(n=150)$ & \\
\hline \multicolumn{6}{|l|}{ Age groups (years) } \\
\hline$\leq 30$ & $0(0)$ & $0(0)$ & $5(3.7)$ & $5(3.3)$ & \multirow[t]{3}{*}{0.907} \\
\hline $31-50$ & $8(66.7)$ & $2(50)$ & $74(55.2)$ & $84(56)$ & \\
\hline$>50$ & $4(33.3)$ & $2(50)$ & $55(41)$ & $61(40.7)$ & \\
\hline \multicolumn{6}{|l|}{ Tumor stage } \\
\hline $\mathrm{T} 1$ & $1(8.3)$ & $0(0)$ & $25(18.7)$ & $26(17.3)$ & \multirow[t]{3}{*}{0.690} \\
\hline $\mathrm{T} 2$ & $6(50)$ & $2(50)$ & $71(53)$ & $79(52.7)$ & \\
\hline $\mathrm{T} 3 / \mathrm{T} 4$ & $5(41.7)$ & $2(50)$ & $38(28.4)$ & $45(30)$ & \\
\hline \multicolumn{6}{|l|}{ ki67 index groups (\%) } \\
\hline$\leq 15$ & $3(25)$ & $1(25)$ & $13(9.7)$ & $17(11.3)$ & \multirow[t]{4}{*}{0.272} \\
\hline $16-24$ & $0(0)$ & $0(0)$ & $8(6)$ & $8(5.3)$ & \\
\hline $25-44$ & $1(8.3)$ & $1(25)$ & $43(32.1)$ & $45(30)$ & \\
\hline$>44$ & $8(66.7)$ & $2(50)$ & $70(52.2)$ & $80(53.3)$ & \\
\hline \multicolumn{6}{|l|}{ Nodal status } \\
\hline Positive & $4(33.3)$ & $2(50)$ & $58(43.3)$ & $64(42.7)$ & \multirow[t]{2}{*}{0.763} \\
\hline Negative & $8(66.7)$ & $2(50)$ & $76(56.7)$ & $86(57.3)$ & \\
\hline \multicolumn{6}{|l|}{ Nodal stage } \\
\hline No & $8(66.7)$ & $2(50)$ & $78(58.2)$ & $88(58.7)$ & \multirow[t]{4}{*}{0.232} \\
\hline N1 & $1(8.3)$ & $1(25)$ & $28(20.9)$ & $30(20)$ & \\
\hline N2 & $3(25)$ & $0(0)$ & $10(7.5)$ & $13(8.7)$ & \\
\hline N3 & $0(0)$ & $1(25)$ & $18(13.4)$ & 19 (12.7) & \\
\hline \multicolumn{6}{|l|}{ Histological subtypes } \\
\hline IDC & $9(75)$ & $4(100)$ & $114(85.1)$ & $127(84.7)$ & \multirow[t]{5}{*}{0.419} \\
\hline Papillary & $0(0)$ & $0(0)$ & $6(4.5)$ & $6(4)$ & \\
\hline Medullary & $0(0)$ & $0(0)$ & $1(0.7)$ & $1(0.7)$ & \\
\hline Metaplastic & $2(16.7)$ & $0(0)$ & $12(9)$ & $14(9.3)$ & \\
\hline Mixed & $1(8.3)$ & $0(0)$ & $1(0.7)$ & $2(1.3)$ & \\
\hline \multicolumn{6}{|l|}{ Tumor grade } \\
\hline Grade-I & $0(0)$ & $0(0)$ & $1(0.7)$ & $1(0.7)$ & \multirow[t]{3}{*}{1.000} \\
\hline Grade-II & $1(8.3)$ & $0(0)$ & $18(13.4)$ & $19(12.7)$ & \\
\hline Grade-III & $11(91.7)$ & $4(100)$ & $115(85.8)$ & $130(86.7)$ & \\
\hline \multicolumn{6}{|l|}{ Lymphocytic infiltration } \\
\hline Absent & $0(0)$ & $0(0)$ & $15(11.2)$ & $15(10)$ & \multirow[t]{3}{*}{0.689} \\
\hline Moderate & $9(75)$ & $4(100)$ & $97(72.4)$ & $110(73.3)$ & \\
\hline Severe & $3(25)$ & $0(0)$ & $22(16.4)$ & $25(16.7)$ & \\
\hline \multicolumn{6}{|l|}{ Lymphovascular invasion } \\
\hline Present & $1(8.3)$ & $1(25)$ & $34(25.4)$ & $36(24)$ & \multirow[t]{2}{*}{0.466} \\
\hline Absent & $11(91.7)$ & $3(75)$ & $100(74.6)$ & $114(76)$ & \\
\hline \multicolumn{6}{|l|}{ Dermal lymphatic invasion } \\
\hline Present & $1(8.3)$ & $1(25)$ & $8(6)$ & $10(6.7)$ & \multirow[t]{2}{*}{0.172} \\
\hline Absent & $11(91.7)$ & $3(75)$ & $126(94)$ & $140(93.3)$ & \\
\hline \multicolumn{6}{|l|}{ Type of surgery } \\
\hline Modified radical mastectomy & $10(83.3)$ & $2(50)$ & $82(61.2)$ & $94(62.7)$ & 0.522 \\
\hline $\begin{array}{l}\text { Simple mastectomy with sentinel } \\
\text { lymph node dissection }\end{array}$ & $2(16.7)$ & $2(50)$ & $38(28.4)$ & $42(28)$ & \\
\hline Wide local excision & $0(0)$ & $0(0)$ & $14(10.4)$ & $14(9.3)$ & \\
\hline
\end{tabular}


Table 2 (continued)

\begin{tabular}{|c|c|c|c|c|c|}
\hline & \multicolumn{4}{|l|}{ n (\%) } & \multirow[t]{2}{*}{ P-value } \\
\hline & $\begin{array}{l}\text { No loss } \\
\text { of expression } \\
(n=12)\end{array}$ & $\begin{array}{l}\text { Complete loss } \\
\text { of expression }(n=4)\end{array}$ & $\begin{array}{l}\text { Focal loss } \\
\text { of expression }(n=134)\end{array}$ & Total $(n=150)$ & \\
\hline \multicolumn{6}{|l|}{ Necrosis } \\
\hline Absent & $2(16.7)$ & $1(25)$ & $18(13.4)$ & $21(14)$ & \multirow[t]{3}{*}{0.507} \\
\hline Moderate & $7(58.3)$ & $1(25)$ & $82(61.2)$ & $90(60)$ & \\
\hline Severe & $3(25)$ & $2(50)$ & $34(25.4)$ & $39(26)$ & \\
\hline \multicolumn{6}{|l|}{ Fibrosis } \\
\hline Mild & $4(33.3)$ & $0(0)$ & $38(28.4)$ & $42(28)$ & \multirow[t]{3}{*}{0.167} \\
\hline Moderate & $8(66.7)$ & $2(50)$ & $78(58.2)$ & $88(58.7)$ & \\
\hline Severe & $0(0)$ & $2(50)$ & $18(13.4)$ & $20(13.3)$ & \\
\hline \multicolumn{6}{|c|}{ Insitu component } \\
\hline Present & $2(16.7)$ & $3(75)$ & $53(39.6)$ & $58(38.7)$ & \multirow[t]{2}{*}{0.098} \\
\hline Absent & $10(83.3)$ & $1(25)$ & $81(60.4)$ & $92(61.3)$ & \\
\hline \multicolumn{6}{|c|}{ Pagetoid spread } \\
\hline Present & $0(0)$ & $0(0)$ & $2(1.5)$ & $2(1.3)$ & \multirow[t]{2}{*}{1.000} \\
\hline Absent & $12(100)$ & $4(100)$ & $132(98.5)$ & $148(98.7)$ & \\
\hline \multicolumn{6}{|c|}{ Perinodal extension } \\
\hline Present & $2(16.7)$ & $1(25)$ & $27(20.1)$ & $30(20)$ & \multirow[t]{2}{*}{1.000} \\
\hline Absent & $10(83.3)$ & $3(75)$ & 107 (79.9) & $120(80)$ & \\
\hline
\end{tabular}

Chi Square test applied

P-value $\leq 0.05$, considered as significant

noted, there was loss of membrane reactivity while cytoplasmic staining was retained in few cells. However no significant association was observed between these abnormal expression patterns of cytokeratins with various clinicopathological and prognostic parameters of TNBC in our population. Breast cancer is one of the commonest malignancies in South Asian population especially in young age [9-11].

Gene expression profiling defines basal breast cancers as those exhibiting basal clusters of genes that include EGFR, basal cytokeratin 5/6, C-kit, proliferation cluster and low expression of Her2neu and hormone receptor related genes [12-14]. However basal breast cancer is not a single entity and it includes several subtypes including two basal like subtypes (BL1 and BL2), mesenchymal, mesenchymal stem-like, immunomodulatory and luminal androgen subtypes [15]. In addition to that interferon rich and claudin-low subtypes have also been defined $[16,17]$. Subtyping of basal breast cancers is not recommended by ASCO/CAP. CK 5/6 expression varies from 24 to $72 \%$ in TNBC as reported in previous studies [18, 19]. Other researchers have also proposed prognostic significance of CK 5/6 in node negative breast cancers [20]. Nielson et al. suggested that CK5/6 positive breast cancer have worse prognosis independent of tumor grade, T-stage and hormonal/Her2neu status [21]. Similarly in another study it was proposed that CK5/6 is marker of shorter disease free survival, independent of other prognostic factors of breast cancer [8]. Inanc et al. reported $50.5 \%$ expression of CK5/6 with positive correlation of CK5/6 TNBC with nodal metastasis and tumor size [22]. However, in contrast to these studies we found a low expression of CK5/6 expression in TNBC in our studied population, which may represent a different genomic profile of TNBC in our population, which needs to be explored in further studies. Prognostic role of CK5/6 has been proposed in other cancers of the body [23].

CK8/18 is a luminal cytokeratin mainly expressed in breast epithelium in a membranous and cytoplasmic pattern. Cimpean et al. defined these different patterns of CK8/18 in breast cancer; diffuse cytoplasmic, membranous and combined cytoplasmic and membranous [19]. Some researchers have proposed that loss of CK8/18 expression/low CK8/18 is associated with worse prognosis and high risk of metastasis $[24,25]$. However we did not find any prognostic significance of loss of CK8/18 expression with various prognostic factors and recurrence in TNBC.

We found that $32.7 \%$ of TNBC show focal loss of expression CK8/18. In all these cases there was loss of membranous reactivity of CK8/18 while cytoplasmic staining was retained in few cells. Aiad et al. in a study 
Table 3 Association of CK 5/6 expression with clinicopathologic features of triple negative breast cancer

\begin{tabular}{|c|c|c|c|c|c|}
\hline & \multicolumn{4}{|l|}{ n (\%) } & \multirow[t]{2}{*}{ P-value } \\
\hline & $\begin{array}{l}\text { No loss of expression } \\
(n=94)\end{array}$ & $\begin{array}{l}\text { Complete loss } \\
\text { of expression }(n=7)\end{array}$ & $\begin{array}{l}\text { Focal loss } \\
\text { of expression }(n=49)\end{array}$ & Total $(n=150)$ & \\
\hline \multicolumn{6}{|l|}{ Age groups (years) } \\
\hline$\leq 30$ & $1(1.1)$ & $1(14.3)$ & $3(6.1)$ & $5(3.3)$ & \multirow[t]{3}{*}{0.061} \\
\hline $31-50$ & $49(52.1)$ & $4(57.1)$ & $31(63.3)$ & $84(56)$ & \\
\hline$>50$ & $44(46.8)$ & $2(28.6)$ & 15 (30.6) & $61(40.7)$ & \\
\hline \multicolumn{6}{|l|}{ Tumor stage } \\
\hline $\mathrm{T} 1$ & $20(21.3)$ & $2(28.6)$ & $4(8.2)$ & $26(17.3)$ & \multirow[t]{3}{*}{0.227} \\
\hline $\mathrm{T} 2$ & $47(50)$ & $4(57.1)$ & $28(57.1)$ & $79(52.7)$ & \\
\hline $\mathrm{T} 3 / \mathrm{T} 4$ & $27(28.7)$ & $1(14.3)$ & $17(34.7)$ & $45(30)$ & \\
\hline \multicolumn{6}{|l|}{ ki67 index groups (\%) } \\
\hline$\leq 15$ & $8(8.5)$ & $2(28.6)$ & $7(14.3)$ & $17(11.3)$ & \multirow[t]{4}{*}{0.495} \\
\hline $16-24$ & $6(6.4)$ & $0(0)$ & $2(4.1)$ & $8(5.3)$ & \\
\hline $25-44$ & $32(34)$ & $1(14.3)$ & $12(24.5)$ & $45(30)$ & \\
\hline$>44$ & $48(51.1)$ & $4(57.1)$ & $28(57.1)$ & $80(53.3)$ & \\
\hline \multicolumn{6}{|l|}{ Nodal status } \\
\hline Positive & $40(42.6)$ & $4(57.1)$ & $20(40.8)$ & $64(42.7)$ & \multirow[t]{2}{*}{0.715} \\
\hline Negative & $54(57.4)$ & $3(42.9)$ & $29(59.2)$ & $86(57.3)$ & \\
\hline \multicolumn{6}{|l|}{ Nodal stage } \\
\hline No & $55(58.5)$ & $3(42.9)$ & $30(61.2)$ & $88(58.7)$ & \multirow[t]{4}{*}{0.152} \\
\hline N1 & $16(17)$ & $3(42.9)$ & $11(22.4)$ & $30(20)$ & \\
\hline N2 & $7(7.4)$ & $0(0)$ & $6(12.2)$ & $13(8.7)$ & \\
\hline N3 & $16(17)$ & $1(14.3)$ & $2(4.1)$ & $19(12.7)$ & \\
\hline \multicolumn{6}{|l|}{ Histological subtypes } \\
\hline IDC & $83(88.3)$ & $6(85.7)$ & $38(77.6)$ & $127(84.7)$ & \multirow[t]{5}{*}{0.138} \\
\hline Papillary & $5(5.3)$ & $0(0)$ & $1(2)$ & $6(4)$ & \\
\hline Medullary & $1(1.1)$ & $0(0)$ & $0(0)$ & $1(0.7)$ & \\
\hline Metaplastic & $4(4.3)$ & $1(14.3)$ & $9(18.4)$ & $14(9.3)$ & \\
\hline Mixed & $1(1.1)$ & $0(0)$ & $1(2)$ & $2(1.3)$ & \\
\hline \multicolumn{6}{|l|}{ Tumor grade } \\
\hline Grade-I & $1(1.1)$ & $0(0)$ & $0(0)$ & $1(0.7)$ & \multirow[t]{3}{*}{0.437} \\
\hline Grade-II & $15(16)$ & $0(0)$ & $4(8.2)$ & $19(12.7)$ & \\
\hline Grade-III & $78(83)$ & $7(100)$ & $45(91.8)$ & $130(86.7)$ & \\
\hline \multicolumn{6}{|l|}{ Lymphocytic infiltration } \\
\hline Absent & $9(9.6)$ & $0(0)$ & $6(12.2)$ & $15(10)$ & \multirow[t]{3}{*}{0.585} \\
\hline Moderate & $72(76.6)$ & $5(71.4)$ & $33(67.3)$ & $110(73.3)$ & \\
\hline Severe & $13(13.8)$ & $2(28.6)$ & $10(20.4)$ & $25(16.7)$ & \\
\hline \multicolumn{6}{|l|}{ Lymphovascular invasion } \\
\hline Present & $26(27.7)$ & $2(28.6)$ & $8(16.3)$ & $36(24)$ & \multirow[t]{2}{*}{0.261} \\
\hline Absent & $68(72.3)$ & $5(71.4)$ & $41(83.7)$ & $114(76)$ & \\
\hline \multicolumn{6}{|l|}{ Dermal lymphatic invasion } \\
\hline Present & $7(7.4)$ & $0(0)$ & $3(6.1)$ & $10(6.7)$ & \multirow[t]{2}{*}{1.000} \\
\hline Absent & 87 (92.6) & $7(100)$ & $46(93.9)$ & $140(93.3)$ & \\
\hline \multicolumn{6}{|l|}{ Type of surgery } \\
\hline Modified radical mastectomy & $59(62.8)$ & $4(57.1)$ & $31(63.3)$ & $94(62.7)$ & 0.806 \\
\hline $\begin{array}{l}\text { Simple mastectomy with sentinel } \\
\text { lymph node dissection }\end{array}$ & $25(26.6)$ & $2(28.6)$ & $15(30.6)$ & $42(28)$ & \\
\hline Wide local excision & $10(10.6)$ & $1(14.3)$ & $3(6.1)$ & $14(9.3)$ & \\
\hline
\end{tabular}


Table 3 (continued)

\begin{tabular}{|c|c|c|c|c|c|}
\hline & \multicolumn{4}{|l|}{ n (\%) } & \multirow[t]{2}{*}{ P-value } \\
\hline & $\begin{array}{l}\text { No loss of expression } \\
(n=94)\end{array}$ & $\begin{array}{l}\text { Complete loss } \\
\text { of expression }(n=7)\end{array}$ & $\begin{array}{l}\text { Focal loss } \\
\text { of expression }(n=49)\end{array}$ & Total $(n=150)$ & \\
\hline \multicolumn{6}{|l|}{ Necrosis } \\
\hline Absent & $14(14.9)$ & $1(14.3)$ & $6(12.2)$ & $21(14)$ & \multirow[t]{3}{*}{0.459} \\
\hline Moderate & $58(61.7)$ & $5(71.4)$ & $27(55.1)$ & $90(60)$ & \\
\hline Severe & $22(23.4)$ & $1(14.3)$ & $16(32.7)$ & $39(26)$ & \\
\hline \multicolumn{6}{|l|}{ Fibrosis } \\
\hline Mild & $22(23.4)$ & $2(28.6)$ & $18(36.7)$ & $42(28)$ & \multirow[t]{3}{*}{0.101} \\
\hline Moderate & $58(61.7)$ & $5(71.4)$ & $25(51)$ & $88(58.7)$ & \\
\hline Severe & $14(14.9)$ & $0(0)$ & $6(12.2)$ & $20(13.3)$ & \\
\hline \multicolumn{6}{|c|}{ Insitu component } \\
\hline Present & $42(44.7)$ & $3(42.9)$ & $13(26.5)$ & $58(38.7)$ & \multirow[t]{2}{*}{0.588} \\
\hline Absent & $52(55.3)$ & $4(57.1)$ & $36(73.5)$ & $92(61.3)$ & \\
\hline \multicolumn{6}{|c|}{ Pagetoid spread } \\
\hline Present & $2(2.1)$ & $0(0)$ & $0(0)$ & $2(1.3)$ & \multirow[t]{2}{*}{0.078} \\
\hline Absent & $92(97.9)$ & $7(100)$ & $49(100)$ & $148(98.7)$ & \\
\hline \multicolumn{6}{|c|}{ Perinodal extension } \\
\hline Present & $24(25.5)$ & $1(14.3)$ & $5(10.2)$ & $30(20)$ & \multirow[t]{2}{*}{0.614} \\
\hline Absent & $70(74.5)$ & $6(85.7)$ & $44(89.8)$ & $120(80)$ & \\
\hline
\end{tabular}

P-value

Chi Square test applied

P-value $\leq 0.05$, considered as significant

involving 70 cases of breast cancer found abnormal expression of CK8/18 (cytoplasmic) in 70\% of Egyptian breast cancer, which suggest a lot of heterogeneity in cytokeratin expression of breast cancer in different parts of world [26].

\section{Limitations}

One of the major limitations of our study was only 2 immuno-markers were performed, therefore we suggest a more through IHC and genomic profiling of TNBC in our population for better understanding of this disease in this part of the world. One of the possible reasons of discrepancy found in the results of our study with that of the previously reported literature could be due the fact that tumors generally exhibit a lot of heterogeneity and we performed IHC stains on one representative block. On the other hand; despite standardization, sample under or over fixation and technical issues may also be responsible for these discrepancies.

\section{Additional files}

Additional file 1: Figure S1. CK5/6 and CK 8/18 expression in triple negative breast cancer.

Additional file 2: Figure S2. Kalpien-Meier curve (disease free survival) for CK 8/18 expression in triple negative breast cancer.

\section{Abbreviations}

TNBC: triple negative breast cancer; IHC: immunohistochemistry; ER: estrogen receptor; PR: progesterone receptor; her2neu: human epidermal growth factor receptor 2; CAP: College of American Pathologist.

\section{Authors' contributions}

$\mathrm{AAH}$ and SN: main author of manuscript, have made substantial contributions to conception and design of study. SKH, ZFH, MI, SMA, NF, AK and MME have been involved in requisition, analysis of the data and revision of the manuscript. All authors revise the manuscript. All authors read and approved the final manuscript.

\section{Author details}

${ }^{1}$ Liaquat National Hospital and Medical College, Karachi, Pakistan. ${ }^{2} \mathrm{CMH}$ Institute of Medical Sciences, Multan, Pakistan. ${ }^{3}$ Kandahar University, Kandahar, Afghanistan. ${ }^{4}$ Brown University, Providence, RI, USA.

\section{Acknowledgements}

We gratefully acknowledge all staff members of Pathology, Liaquat National Hospital, Karachi, Pakistan for their help and cooperation.

\section{Competing interests}

The authors declare that they have no competing interests.

Availability of data and materials

Please contact author, Atif Ali Hashmi (doc_atif2005@yahoo.com) for data requests.

\section{Consent to publish}

Not applicable.

\section{Ethical approval and consent to participate}

Ethics committee of Liaquat National Hospital, Karachi, Pakistan approved the study. Written informed consent was obtained from the patients for the participation. 


\section{Funding}

There was no funding available for this manuscript.

\section{Publisher's Note}

Springer Nature remains neutral with regard to jurisdictional claims in published maps and institutional affiliations.

Received: 2 April 2018 Accepted: 4 June 2018

Published online: 08 June 2018

\section{References}

1. Swain S. Triple-negative breast cancer: metastatic risk and role of platinum agents 2008 ASCO clinical science Symposium, 2008. June 3; 2008.

2. Hashmi AA, Edhi MM, Naqvi H, Khurshid A, Faridi N. Molecular subtypes of breast cancer in South Asian population by immunohistochemical profile and Her2neu gene amplification by FISH technique: association with other clinicopathologic parameters. Breast J. 2014;20(6):578-85.

3. Hashmi AA, Edhi MM, Naqvi H, Faridi N, Khurshid A, Khan M. Clinicopathologic features of triple negative breast cancers: an experience from Pakistan. Diagn Pathol. 2014:28(9):43.

4. Hammond ME, Hayes DF, Dowsett M, et al. American Society of Clinical Oncology/College of American Pathologists guideline recommendations for immunohistochemical testing of estrogen and progesterone receptors in breast cancer (unabridged version). Arch Pathol Lab Med. 2010;134:e48.

5. Wolff AC, Hammond ME, Hicks DG, et al. Recommendations for human epidermal growth factor receptor 2 testing in breast cancer: American Society of Clinical Oncology/College of American Pathologists clinical practice guideline update. J Clin Oncol. 2013;31:3997.

6. Shah SP, Roth A, Goya R, et al. The clonal and mutational evolution spec trum of primary triple-negative breast cancers. Nature. 2012;486:395.

7. Cheang MC, Voduc D, Bajdik C, Leung S, McKinney S, Chia SK, Perou CM, Nielsen TO. Basal-like breast cancer defined by five biomarkers has superior prognostic value than triple-negative phenotype. Clin Cancer Res. 2008;14:1368-76.

8. Abd El-Rehim DM, Pinder SE, Paish CE, Bell J, Blamey RW, Robertson JF, Nicholson RI, Ellis 1O. Expression of luminal and basal cytokeratins in human breast carcinoma. J Pathol. 2004;203(2):661-71.

9. Haroon S, Hashmi AA, Khurshid A, Kanpurwala MA, Mujtuba S, Malik B, Faridi N. Ki67 index in breast cancer: correlation with other prognostic markers and potential in pakistani patients. Asian Pac J Cancer Prev. 2013:14(7):4353-8

10. Hashmi AA, Faridi N, Khurshid A, Naqvi H, Malik B, Malik FR, Fida Z, Mujtuba S. Accuracy of frozen section analysis of sentinel lymph nodes for the detection of Asian breast cancer micrometastasis_experience from Pakistan. Asian Pac J Cancer Prev. 2013:14(4):2657-62.

11. Hashmi AA, Aijaz S, Khan SM, Mahboob R, Irfan M, Zafar NI, Nisar M, Siddiqui M, Edhi MM, Faridi N, Khan A. Prognostic parameters of luminal A and luminal B intrinsic breast cancer subtypes of Pakistani patients. World J Surg Oncol. 2018;16(1):1.
12. Livasy CA, Karaca G, Nanda R, et al. Phenotypic evaluation of the basallike subtype of invasive breast carcinoma. Mod Pathol. 2006;19:264.

13. Carey LA, Rugo HS, Marcom IW, et al. TBCRC 001: EGFR inhibition with cetuximab added to carboplatin in metastatic triple-negative (basallike) breast cancer (abstract 1009). J Clin Oncol. 2008:26:1009.

14. Nielsen TO, Hsu FD, Jensen $\mathrm{K}$, et al. Immunohistochemical and clinical characterization of the basal-like subtype of invasive breast carcinoma. Clin Cancer Res. 2004;10:5367.

15. Lehmann BD, Bauer JA, Chen X, et al. Identification of human triplenegative breast cancer subtypes and preclinical models for selection of targeted therapies. J Clin Invest. 2011;121:2750.

16. Teschendorff AE, Miremadi A, Pinder SE, et al. An immune response gene expression module identifies a good prognosis subtype in estrogen receptor negative breast cancer. Genome Biol. 2007;8:R157.

17. Prat A, Parker JS, Karginova O, et al. Phenotypic and molecular characterization of the claudin-low intrinsic subtype of breast cancer. Breast Cancer Res. 2010;12:R68.

18. Ryu DW, Jung MJ, Choi WS, Lee $\mathrm{CH}$. Clinical significance of morphologic characteristics in triple-negative breast cancer. J Korean Surg Soc. 2011:80(5):301-6.

19. Dogu GG, Ozkan M, Ozturk F, Dikilitas M, Er O, Ozturk A. Triple-negative breast cancer: immunohistochemical correlation with basaloid markers and prognostic value of survivin. Med Oncol. 2010;27(1):34-9.

20. van de Rijn $M$, Perou $C M$, Tibshirani $R$, Haas $P$, Kallioniemi $O$, Kononen J, Torhorst J, Sauter G, Zuber M, Köchli OR, Mross F, Dieterich H, Seitz R, Ross D, Botstein D, Brown P. Expression of cytokeratins 17 and 5 identifies a group of breast carcinomas with poor clinical outcome. Am J Pathol. 2002;161(6):1991-6

21. Nielsen TO, Hsu FD, Jensen K, Cheang M, Karaca G, Hu Z, HernandezBoussard T, Livasy C, Cowan D, Dressler L, Akslen LA, Ragaz J, Gown AM, Gilks CB, van de Rijn M, Perou CM. Immunohistochemical and clinical characterization of the basal-like subtype of invasive breast carcinoma. Clin Cancer Res. 2004;10(16):5367-74.

22. Inanc M, Ozkan M, Karaca H, Berk V, Bozkurt O, Duran AO, Ozaslan E, Akgun H, Tekelioglu F, Elmali F. Cytokeratin 5/6, c-Met expressions, and PTEN loss prognostic indicators in triple-negative breast cancer. Med Oncol. 2014;31(1):801

23. Hashmi AA, Hussain ZF, Irfan M, Edhi MM, Kanwal S, Faridi N, Khan A. Cytokeratin 5/6 expression in bladder cancer: association with clinicopathologic parameters and prognosis. BMC Res Notes. 2018;11(1):207.

24. Becker $M$, et al. Sensitive PCR method for the detection and real-time quantification of human cells in xenotransplantation systems. $\mathrm{Br} J$ Cancer. 2002;87(11):1328-35. https://doi.org/10.1038/sj.bjc.6600573.

25. Schaller $\mathrm{G}$, et al. Elevated keratin 18 protein expression indicates a favorable prognosis in patients with breast cancer. Clin Cancer Res. 1996;2(11):1879-85

26. Aiad HA, Samaka RM, Asaad NY, Kandil MA, Shehata MA, Miligy IM. Relationship of CK8/18 expression pattern to breast cancer immunohistochemical subtyping in Egyptian patients. Ecancermedicalscience. 2014;20(8):404

Ready to submit your research? Choose BMC and benefit from

- fast, convenient online submission

- thorough peer review by experienced researchers in your field

- rapid publication on acceptance

- support for research data, including large and complex data types

- gold Open Access which fosters wider collaboration and increased citations

- maximum visibility for your research: over 100M website views per year

At BMC, research is always in progress.

Learn more biomedcentral.com/submissions 